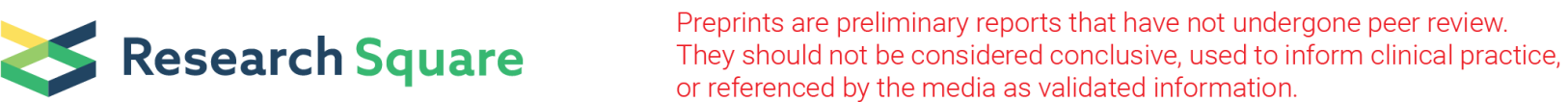

\section{Efficacy and safety of post-scleral injection of triamcinolone acetonide for macular edema in branch retinal vein occlusion: 3 month results from a prospective randomized, controlled and open label clinical trial}

Hongxuan Lie

Changhai Hospital

Meidong Zhu

The University of Sydney Save Sight Institute

Sha Li

Army Medical University

Li Ran

Army Medical University

Xiaohong Meng

Army Medical University

Yanlin Long

Army Medical University

Yong Liu

Army Medical University

Yi Wang

Aier Eye Hospital Group

Shiying Li ( $\nabla$ shiying_li@126.com )

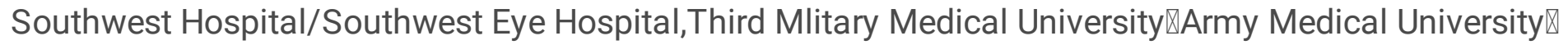
https://orcid.org/0000-0001-9783-9520

Research article

Keywords: Branch retinal vein occlusion, Post-scleral injection, Triamcinolone acetonide, Ranibizumab

Posted Date: February 20th, 2020

DOl: https://doi.org/10.21203/rs.2.24134/v1 
License: (c) (i) This work is licensed under a Creative Commons Attribution 4.0 International License. Read Full License 


\section{Abstract}

Background: To assess the safety and efficacy of post-scleral injection of triamcinolone acetonide (TA) in macular region to treat macular edema secondary to branch retinal vein occlusion (BRVO), and compare it with intravitreal injection of TA and Ranibizumab. Methods: This is a prospective, double masked and single-center study. Thirty-six eyes, one from each participant who was diagnosed with macular edema secondary to BRVO between July 2011 and December 2014, were randomised into three groups to receive post-sclera injection of $20 \mathrm{mg} / 0.05 \mathrm{ml} \mathrm{TA}$ (PS-TA), intravitreal injection of $2 \mathrm{mg}$ TA (IV-TA) and intravitreal injection of $0.5 \mathrm{mg}$ Ranibuzmab (IV-Ranibizumab), respectively. Changes of retinal thickness in optical coherence tomography (OCT) in foveal (zone 1) and average macular area (1-9 zones) at month 1 and 3 from baseline were the primary outcomes. The secondary study outcomes include changes of best corrected visual acuity (BCVA) and intraocular pressure (IOP) at month 1 and 3 from baseline and profile of adverse events. Results: The mean thickness changes from the baseline in macular fovea (zone 1) were statistically significant at month 1 in IV-TA and PS-TA groups, respectively (P-value $<0.0005$ and $P$-value $=0.032)$, but not in Ranibizumab group $(P$-value $=0.083)$. At month 3 , the mean thickness change from the baseline in macular fovea (zone 1) was statistically significant only in IV-TA group (P-value $=0.001)$. There was no significant difference in the mean thickness changes in macular region (zone 1-9) from baseline to month 1 or 3 in any group. No statistically significant differences were detected across the three treatment groups in mean thickness changes in macular fovea (zone 1) nor macular region (zone 1-9), over 1 and 3 months from baseline (P-values > 0.05). No Serious Adverse Event was detected in any treatment group during the study period. Two eyes in the IV-TA group had mild elevated IOP after the injection. Conclusion: PS-TA injection near macular region is safe and effective in treating macular edema secondary to BRVO. PS-TA could reduce the incidence of intraocular hypertension significantly, and thus has the advantages of safety compared to IVTA.

\section{Background}

Retinal vein occlusion (RVO) is the second most common cause of retinal vascular abnormality after diabetic retinopathy [1]. Eyes suffering from this disease are vulnerable to vision loss and may become blind due to its complications. According to where the occlusion is located, RVO is classified into central retinal vein occlusion (CRVO), hemi-retinal vein occlusion (HRVO), and branch retinal vein occlusion (BRVO). It has been reported that the prevalence of BRVO is $0.6-1.6 \%$ in group of age 65 to 74 years old, while the incidence is about 2.14/1000 per year in the population over 40 years old [2]. BRVO often occur at arteriovenous crossings, and the artery is anterior to the vein at the occlusion in most cases. The degree of macula involvement determines the level of vision loss, which may be caused by ischemic injury and/or secondary macular edema. Macular edema is commonly seen in BRVO patients with 5$15 \%$ incidence rate within the first year of the disease course and is one of the most significant causes of central vision loss in these patients $[3,4]$.

The first line treatment of macular oedema is intravitreal injection of anti-vascular endothelial growth factor (VEGF) [5, 6]; however, it may increase the risks of stroke and intraocular infection [7]. In addition, 
the cost of intravitreal anti-VEGF such as Ranibizumab may create burdens for the patients and social health system due to its high price [8-10], particularly in developing countries. Triamcinolone acetonide (TA) is an approved effective treatment for macular oedema secondary to BRVO. However, TA has high profile in intraocular hypertension and cataract formation [11-14]. These complications are does and time dependant [15]. To minumise the adverse events of elevation of intraocular pressure and cataract formation, we developed the patent set of post-sclera injection for TA delivery (patent number ZL 20132 0740202.0). This treatment has direct effect to macular edema. The instrument allows accurate postscleral injection with small dosage of TA and hydrotropic substance and solutes carrying positive charge would penetrate the sclera more easily $[16,17]$. This randomized controlled clinical trial is aimed to assess the safety and efficacy of post-sclera injection of TA in macular region in treatment of BRVO with macular edema, comparing with conventional intravitreal injections of TA and Ranibizumab.

\section{Methods}

\subsection{Study design}

A prospective, double-masked and open label randomized clinical trial was conducted on 36 participants attending the ophthalmology clinic of Southwest Hospital, China, between 2011 and 2014. All patients were followed up to 3 months when possible. The study has been approved by the Ethics Committee of Southwest Hospital, Third Military Medical University, and registered in Chinese Clinical Trial Registration Center (registration number: ChiCTR-OPC-15005910) before study commencement. Informed consent was obtained from all participants before enrollment.

\subsection{Research subjects}

Thirty-six participants, diagnosed with BRVO with macular edema in the ophthalmology clinic of Southwest Hospital, China during 2011-2014, were included in the study. Only one eye was recruided as a study eye from each patient. If both eyes of the participant were meeting the criteria, the eye with poorer vision was slected as a study eye. Examinations including BCVA, IOP, fundus color photography, fundus autofluorescence (FAF), fundus fluorescein angiography (FFA), and OCT confirmed that all the patients met the diagnostic criteria of BRVO with macular edema.

Inclusion criteria were as follows: male or female participants; age $\geq 18$ years old; macular edema secondary to BRVO involving the fovea within 12 months of BCVA diagnosis at the screening visit in study eye; central macular thickness (CMT) $>250 \mu$ measured by OCT and BCVA between 19 and 73 letters in Early Treatment Diabetic Retinopathy Study (ETDRS) charts (equivalent to 20/400 - 20/40 Snellen acuity); fellow eye with BCVA > 34 ETDRS chart letters (equivalent to $>20 / 200$ Snellen acuity.

Exclusion criteria were: uncontrolled IOP of more than $25 \mathrm{mmHg}$; significant corneal or lenticular opacities, which would affect the assessment of treatment effect and vision improvement; previous intraocular surgery history; the patients take other investigational drugs; intravitreal anti-VEGF treatment within 6 weeks prior to screen visit; the participants were treated with corticosteroid hormone systemically 
or locally for more than 30 days continiouly within 6 months at the screening visit; the participants were treated with corticosteroid hormone in or around the eyes within 3 months at the screening visit; the particpants received full-retina laser photocoagulation 3 months prior to the screen; myocardial infarction, transient ischemic attack, or cerebrovascular accident within 90 days prior to screen visit; and current pregnancy or lactation. All ocular exclusion criteria refer to the study eye only.

\subsection{Randomized grouping and therapeutic methods}

Participants who were eligible to the study were uniquely numbered from 1-36 according to the sequence of enrollment and randomized into three groups to receive one of the three treatments: intravitreal injection of TA (IV-TA), intravitreal injection of Ranibizumab (IV-Ranibizumab) and post-sclera injection of TA in macular region (PS-TA).

Patients in PS-TA group received the treatemnt via patented post-sclera injection needle set and injection method (China's National Utility Model Patent; number ZL 20132 0740202.0). The instrument set includes a needle and connector, and the needle is a blunt angled needle with a radian similar to that of the eyeball.

During the procedure, the participants received a topical anesthesia (benoxinate hydrochloride $0.4 \%$ ) and followed by a topical antibiotic drops (chloramphenicol solution $0.5 \%$ ). The needle was connected to an ordinary syringe and was inserted through a small incision at the temporal conjunctiva $6 \mathrm{~mm}$ from the corneal limbus. The needle was slided in on the surface of the sclera with the tip reaching macular region. Post-sclera injection of $20 \mathrm{mg}$ in $0.5 \mathrm{ml}$ TA was performed. Daily B-ultrasonography was conducted in the first 6 days after the injection to observe drug residue posteriorly to the macula.

Injection of IV-TA and IV-Ranibizumab were performed as an outpatient procedure in procedure rooms with strict sterile conditions. After topical anesthesia with $0.4 \%$ benoxinate hydrochloride eye drops, the ocular surface and the lid were disinfected with povidone-iodine. Intravitreal injection was carried out using a 30-gauge needle at $3.5 \mathrm{~mm}$ posterior to the limbus (TA $2 \mathrm{mg} / 0.05 \mathrm{ml}$ or Ranibizumab $0.3 \mathrm{mg} / 0.05 \mathrm{ml}$ ). The injection site was compressed by cotton swab to avoid reflux, and the fundus was examined to rule out any complication and to check perfusion of the retinal artery.

\subsection{Outcomes assessment}

The primary efficacy outcome was the changes of retinal thickness in OCT (Topcon, 3D, Japan) in foveal area (zone 1) and macular area (1-9 zones) at 1 and 3 month compared to baseline. The secondary study outcomes include changes of BCVA and IOP (Cannon, TX-F, Japan) at 1 and 3 month from baseline and the profile of adverse events.

Participant's retina of study eye was scanned along 8 meridian lines with a scan length of $6 \mathrm{~mm}$. The distance (thickness) between the inner surface of the retina at the macular fovea and the light band of retinal pigment epithelium and choroid blood capillary was measured. The average thickness of 1-9 zones is the mean value of the thickness of the 9 zones in macular area. The 9 zones are centered at the 
macular fovea and numbered from 1-9. 1 represents the macular fovea (zone 1 with a diameter of $1000 \mu \mathrm{m}$ ); 2-5 represent the inner ring (diameter: 1000-3000 $\mu \mathrm{m}$ ), including 4 equal zones: temporal side, upper side, nasal side, and lower side; 6-9 represent the outer ring (diameter: 3000-6000 $\mu \mathrm{m}$ ), including 4 equal zones: temporal side, upper side, nasal side, and lower side (see Fig. 1A). The secondary efficacy observation outcome included BCVA, which was examined using ETDRS visual acuity chart (Precision Vision, 2425E, USA). The safety outcome include profile of advese event, serious adverse events and IOP changes. The IOP was measured using non-contact tonometer(Cannon, TX-F, Japan) in each visit.

\subsection{Statistical method}

For each of three treatments considered in this study, therapeutic effect on thickness in macular fovea (zone 1; also known as CMT), as well as on average thickness of whole macular area (zone 1-9) at month 1 and 3 separately, was evaluated by comparing the thickness before and after applying the treatment using paired $t$ test. Analysis of Variance (ANOVA) when appropriate, or its Welch alternative for unequal variances [18], was used for comparing change, over 1 and 3 months respectively, in thickness in macular fovea (zone 1), average thickness in macular area (1-9 zones), BCVA and IOP across the three treatments studied. Treatment effects were further investigated using generalised estimating equations [19] that accounted for possible correlation in repeated measurements over time while adjusting for other factors or potential confounders in the study.

Analyses presented here were performed using SPSS statistical software (version 23; IBM Corp, Armonk, NY, USA). A P-value of less than 0.05 was considered statistically significant (ie, using $5 \%$ significance level) in all analyses presented in this paper.

\section{Results}

\subsection{Patient baseline information}

Thirty-six eyes from 36 participants with macular edema secondary to BRVO were included in this study. Month 1 and month 3 post injection follow-ups were completed on 30 of the 36 participants. Four participants in PS-TA group and 2 patients in IV-TA group had month 1 post-injection visit only. The participants include 15 males and 21 females, age ranged from 39 to 78 years old (mean \pm standard deviation: $60.0 \pm 10.3)$.

There was no significant difference across the three treatment groups at baseline in thickness in macular fovea (zone 1) $(P$-value $=0.307)$ nor in average thickness in macular area $(1-9$ zones $)(P$-value $=0.236)$. The three treatment groups had no significant difference in mean BCVA at baseline ( $P$-value $=0.282)$ although Ranibizumab group appeared to have relatively lower average BCVA (IV-TA: 40.2 24.7 , IVRanibizumab: $24.8 \pm 26.3$, PS-TA: $39.8 \pm 29.1$, P-value $=0.282$ ) as shown in Table 1. Furthermore, the IOPs of all the patients were within normal value range of $12.4 \mathrm{mmHg}$ to $19.7 \mathrm{mmHg}$. 


\subsection{Post injection thickness changes}

\subsubsection{Intragroup difference of thickness changes}

The mean thickness changes (ie, reduction) at month 1 from baseline in macular fovea (zone 1) and macular area (1-9 zones) were 78.6 and 20.7 for PS-TA, 236.3 and 45.3 for IV-TA and 145.8 and 30.0 for ranibizumab as shown in Table 2. Such reduction in CMT (zone 1) was only considered significant for patients treated with PS-TA (P-value $=0.032)$ and IV-TA $(P$-value $<0.0005)$, but not for those treated with ranibizumab (P-value $=0.083)$. (Fig. 2).

\subsubsection{Intergroup difference of thickness changes in macular fovea (zone 1) and average thickness} changes in macular area (1-9 zones)

As shown in Table 3, IV-TA group appeared to have the most significant reduction in CMT and mean CMT at month 1 compared to other treatment groups, however, the changes in both CMT and mean CMT across the three treatments were not statistically significant (P-values $=0.133$ and 0.730 , respectively). No significant difference was detected neither in mean change in CMT (zone 1) or mean CMT (zone1-9) at month 3 from the baseline in three groups ( $P$-values $=0.629$ and 0.941 , respectively). Furthermore, CMT generaly bounced back noticeably from month 1 to month 3 although not statistically significant, while mean CMT did not change from month 1 to 3 . (Fig. 3).

The treatemt effect on CMT was further investigated using more sophiscated statistical analysis via GEE method, accounting for possible correlation among repeated measurements over time (month 0,1 and 3) and also other possible confounding factors, such as age and gender. The results from the analysis are similar to those from ANOVA described above, confirming CMT decreased from the baseline to month 1 with not significant further change from month 1 to month 3 , and there was no significant treatment difference on CMT even after adjusted for possible confounding effects from age, gender and baseline BCVA. Similar analysis was also carried out for mean CMT, confirming no significant difference in mean CMT across the three treatments suggested by the ANOVA earlier. However, a significant difference in mean CMT over time (P-value $=0.022)$ was detedcted after accounting for correlation among repeated measurements and adjusting for gender, age and baseline BCVA, unlike the findings on mean CMT from the ANOVA. The mean CMT was reduced from the baseline to month 1 with no further change to month 3.

\subsection{BCVA changes after injection}

For each treatment group, average change in BCVA was examined. As shown in Table 4, no significant change was detected either at month 1 or month 3 for PS-TA group. BCVA was improved by 15.4 letters on average over the first month after the treatment for IV-TA group (P-value < 0.0005$)$, however, further improvement from month 1 to 3 was marginal (P-value $=0.058)$. Similar but slightly less improvement in BCVA was also observed in IV-Ranibizumab group at month 1 and month 3 ( $P$-value=0.034). (Table.5) 
Changes in BCVA at month 1 and 3 were also compared across the three treatments respectively. There was no treatment difference in the changes even after accounting for patients' baseline factors (P-values $>0.05)$. However BCVA change over time was confirmed by the GEE analysis ( $P$-value $<0.0005)$, and baseline BCVA was associated with BCVA change over time among the three groups ( $P$-value $=0.001)$.

\subsection{TA Pharmacokinetics in PS-TA group}

TA was seen in post-sclera closeing to macular area in the first 5 days after the injection, and the residue amount gradually diminished with time. On the $6^{\text {th }}$ day after the injection, no sound shadow was observed posteriorly to the wall of eyeball, indicating the drug had been absorbed (see Fig. 1B).

\subsection{Advense events (AEs)}

The IOPs in three groups were within normal value range at month 1 post-injecton (IV-TA: 18.9 \pm 2.7 , IVRanibizumab: $16.1 \pm 1.8$, PS-TA:16.2 \pm 2.1 ) and month 3 (IV-TA: 17.3 \pm 2.1 , IV-Ranibizumab: 16.2 \pm 1.9, PSTA:16.5 \pm 2.0$)$ except the two patients.

No SAE was detected during follow-up period. AEs such as conjunctival hemorrhage, retinal exudation, retinal hemorrhage, and infections were not found in all 36 patients. Two patients in IV-TA group showed IOP elevation at month 1 post-injection $(25.9 \mathrm{mmHg}$ and $22.7 \mathrm{mmHg}$ ) but recovered to 21.5 and 19.1 $\mathrm{mmHg}$ at month 3 without treatment. All participants had stable blood pressure and blood sugar levels during observed period.

\section{Discussion}

Macular edema caused by BRVO is a major cause of vision loss, and there is no effective oral medication to treat for the time being [20]. Currently, the first line treatment of macular oedema secondary to BCVO is intravitreal injection anti-VEGF, Howevr, it has some limitations and disadvantages: intravitreal injection of anti-VEGF has the risk of intraocular infection [21] and also creates burdens to patients due to its costs and frequent visit to clinics, especially in developing countries. Tramcinolone has been confirmed to be effective for the treatment of BRVO with macular edema because of its potent antipermeability and antiinflammatory properties $[15,22]$. However, complications resulting from intravitreal injection of TA therapy include cataract formation (24.2\%), elevation of intraocular pressure (20\%), infectious endophthalmitis, noninfectious endophthalmitis, and retinal detachment [23]. Retrobulbar injection or post-Tenon's capsule injection TA has the risk of piercing the eyeball [24]; peribulbar injection of TA yields poor outcomes. This study explores a safe, economic, and effective treatment of BRVO with macular edema by a prospective randomized, controlled clinical trial between conventional treatment and our new technique.

We use a patented method of post-scleral injection of TA to treat macular edema secondary to BRVO. The instrument and method for post-sclera injection described in this study are different from the instrument and method for post-Tenon's capsule injection. In the latter, drugs are injected into the retrobulbar Tenon's 
capsule, which is relatively far from macular area, therefore the efficacy is poor. However if the injection is too close to the macula, the risks of piercing the eyeball and damage inferior rectus muscle would be increased. The outer surface of the sclera is covered by the loose episcleral fascia, which mainly consists of collagenous fiber embedded with glycosaminoglycan substrate, and the post-sclera collagenous fiber is looser than the ante-sclera part [25]. Therefore, peri-post-scleral injection is beneficial to drug accumulation and absorption. To reach the retinal neurepithelium layer, a drug has to panetrate through several membranes including sclera, choroid, Bruch membrane, and retinal pigment epithelium. Experimental results show that the largest molecular weight of substances, to which the sclera is permeable, is $150 \mathrm{KDa}$ [26]. TA, whose molecular weight is $434.5 \mathrm{Da}$, is very easy to pass through it. In our study, a daily B-ultrasonography was performed continuously to monitor TA diminish after post-scleral injection. TA was seen posteriorly to the macula in the first 5 days after the injection, and the residue amount gradually diminished with time. On the 6th day after the injection, no sound shadow was observed posteriorly to the wall of eyeball, indicating the drug had been absorbed fully and TA could exist behind the sclera in macular area for 5 days.

The peri-post-scleral injection is easy to learn and operate. Because vitreous cavity is not involved in this procedure, the risks of intraocular infection could be avoid. Further more as the needle is blunt, the risk of eyeball punctuation is reduced. Inserting the needle through the small incision with minimal trauma at the conjunctiva above the temporal can avoid damage extraocular muscle. In addition, because drugs are directly injected into the post-macula region, the episcleral Tenon's capsule can retain local drug concentration and yield direct effect in curing macular edema. This method also avoids significant AE of IOP elevation that IV-TA usually casued. The study indicated that all the study eyes in PS-TA group had no significant changes of IOP post treatment.

TA is cost-effective and easier to obtain in developing countried such as China. Furthermore, TA can also stabilize vascular endothelial barrier by reducing the release of prostaglandin through arachidonic acid pathway [27]. It reduce inflammation by suppressing inflammatory cell proliferation and migration and by decreasing the synthesis and release of proinflammatory molecules (prostaglandins, leukotrienes, vascular endothelial growth factor, and intercellular adhesion molecule 1) [28].

This study found that the differences of thickness changes in macular fovea (zone 1) and mean thickness change in macular area (1-9 zones) between PS-TA group and IV-Ranibizumab and IV-TA groups at month 1 and 3 were not statistically significant, indicating that the effect of PS-TA group was samilar to that of Ranibizumab. This lack of significance for ranibizumab effect on CMT over the first month might be due to the surprisedingly large variability $(S D=265.1)$ among the 12 patients in this treatment group.

The study found that mean CMT in month 3 at zone 1 was relapsed in IV-TA group $(P=0.017)$ while PSTA group maintained low mean CMT during whole treatment period. Though the effect in IV-TA group was better than that in PS-TA group at early stage (month 1 post-injection, $\mathrm{P}<0.01$ ), the advantage was 
obviously weakened 3 months after the injection and PS-TA group outmatched IV-TA group in effective duration.

This study found that no significant change in BCVA was detected at month 1 for PS-TA group while the mean thickness changes at month 1 from baseline in macular fovea (zone 1$)$ was significant (P-value = 0.032 ). This result might be largely due to the a poor correlation between morphological changes and functional outcomes. The same phenomenon occurred in IV-Ranibizumab group.

By the GEE analysis result, we showed that PS-TA injection is as effective as IV-TA injection and IVRanibizumab injection in BCVA improvement and CMT reduction for macular edema secondary to BRVO.

Nonetheless, in advense events the use of PS-TA for BRVO offers significant advantages over IV-TA because IOP elevation and cataract formation were not observed in patients treated with PS-TA.

The limitation of this study is that the follow-up period is short. In addition, the small sample size may reduce the power to detect the difference of efficacy between the treatment groups.

\section{Conclusion}

This study indicates that with our patented instrument and methods, PS-TA can be used to treat macular edema secondary to BRVO; the effect of PS-TA is stable during the follow-up period up to 3 months, and there was no statistical difference in the effects among PS-TA, IV-TAand IV-Ranibizumab. PS-TA is cost effective and safe. Therefore in the situation where anti-VEGF angent is not available, PS-TA may be used as a substitute for BRVO treatment.

\section{Abbreviations}

TA:Triamcinolone Acetonide; BRVO:Branch retinal vein occlusion; CRVO:Central retinal vein occlusion; HRVO:Hemi-retinal vein occlusion; RVO:Retinal vein occlusion; PS-TA:Post-sclera injection of TA; IVTA:Intravitreal injection of TA; IV- Ranibizumab:Intravitreal injection of Ranibuzmab; OCT:Optical coherence tomography; BCVA:Best corrected visual acuity; IOP:Intraocular pressure; VEGF:Vascular endothelial growth factor; FAF:Fundus autofluorescence; FFA:Fundus fluorescein angiography; CMT:Central macular thickness; ETDRS:Early Treatment Diabetic Retinopathy Study; ANOVA:Analysis of Variance; AEs:Advense events;

\section{Declarations}

\section{Ethics approval and consent to participate}

The study has been approved by the Ethics Committee of Southwest Hospital, Third Military Medical University, and registered in Chinese Clinical Trial Registration Center (registration number: ChiCTR-OPC15005910) before study commencement. All included patients signed an informed written consent. 
Not applicable

\section{Availability of data and material}

Available upon request from the authors.

\section{Competing interests}

The authors declare that they have no competing interests.

\section{Funding}

National Nature Science Foundation of China (81974138): design of the study.

Chongqing Social and livelihood science innovation grant (cstc2017shmsA130100): collection of data.

National Basic Research Program of China(2018YFA0107301): analysis, and interpretation of data.

Third Military Medical University (Army Medical University) research grant(2017XYY02): writing the manuscript.

\section{Authors' contributions}

SYL: the idea and concept of the study, collection of data, carried out therapy. HXL: writing the manuscript, collecting data, analysis of the data. MDZ: revised and edited the manuscript, revised the statistical analysis. SL: reviewing article, revising the statistical analysis. LR, XHM, YLL, YL, YW: shared in writing manuscript, analysis of data, performed the examiexaminations, shared in idea of the study. All authors contributed to the drafting, critical revision and final approval of the manuscript.

\section{Acknowledgements}

We are grateful to Dr Kehui Luo, Department of Mathematics and Statistics, Faculty of Science and Engineering, Macquarie University, Sydney, Australia. Dr Zengwei Zhao, Third Military Medical University (Army Medial University), Chongqing, China, for their help in clinical and statistiacal data analysis. We thank technicians Minfang Zhang, Sha Li, Cheng Sun. We also thank all the collaborators of Southwest Hospital/Southwest Eye Hospital, Third Military Medical University (Army Medial University), Chongqing, China.

\section{References}

1. Lam F C, Chia S N, Lee R M. Macular grid laser photocoagulation for branch retinal vein occlusion. Cochrane Database Syst Rev. 2015;CD008732.

2. Klein R, Klein B E, Moss S E, Meuer S M. The epidemiology of retinal vein occlusion: the Beaver Dam Eye Study. Trans Am Ophthalmol Soc. 2000;98:133-41; discussion 141-3. 
3. Laouri M, Chen E, Looman M, Gallagher M. The burden of disease of retinal vein occlusion: review of the literature. Eye (Lond). 2011;25:981-8.

4. Moradian S, Faghihi H, Sadeghi B, Piri N, Ahmadieh H, Soheilian M, Dehghan M H, Azarmina M, Esfahani M R. Intravitreal bevacizumab vs. sham treatment in acute branch retinal vein occlusion with macular edema: results at 3 months (Report 1). Graefes Arch Clin Exp Ophthalmol. 2011;249:193-200.

5. Ip M S, Gottlieb J L, Kahana A, Scott I U, Altaweel M M, Blodi B A, Gangnon R E, Puliafito C A. Intravitreal triamcinolone for the treatment of macular edema associated with central retinal vein occlusion. Arch Ophthalmol. 2004;122:1131-6.

6. Saraiva V S, Sallum J M, Farah M E. Treatment of cystoid macular edema related to retinitis pigmentosa with intravitreal triamcinolone acetonide. Ophthalmic Surg Lasers Imaging. 2003;34:398-400.

7. Irini C, Luke N, Sobha S, ME F. Intravitreal steroid and anti-vascular endothelial growth agents for the management of retinal vein occlusion: evidence from randomized trials. Expert Opin Biol Ther. 2015;15:1685-97.

8. Mylonas G, Sacu S, Dunavoelgyi R, Matt G, Blum R, Buehl W, Pruente C, Schmidt-Erfurth U. Response of retinal sensitivity to ranibizumab treatment of macular edema after acute branch retinal vein occlusion. Retina. 2013;33:1220-6.

9. Jonas J B. Intravitreal triamcinolone acetonide: a change in a paradigm. Ophthalmic Res. 2006;38:218-45.

10. Martidis A, Duker J S, Greenberg P B, Rogers A H, Puliafito C A, Reichel E, Baumal C. Intravitreal triamcinolone for refractory diabetic macular edema. Ophthalmology. 2002;109:920-7.

11. Bakri S, Beer P. The effect of intravitreal triamcinolone acetonide on intraocular pressure. Ophthalmic Surg Lasers Imaging. 2003;34:386-90.

12. Jonas J, Kreissig I, Degenring R. Intraocular pressure after intravitreal injection of triamcinolone acetonide. Br J Ophthalmol. 2003;87:

13. Jonas J, Akkoyun I, Kamppeter B, Kreissig I, Degenring R. Branch retinal vein occlusion treated by intravitreal triamcinolone acetonide. Eye 2005;19:

14. Cekiç O, Chang S, Tseng J, Barile G, Priore L D, Weissman H, Schiff W, Ober M. Intravitreal triamcinolone injection for treatment of macular edema secondary to branch retinal vein occlusion. Retina. 2005;25:

15. Chen $\mathrm{C}$, Chen $\mathrm{Y}, \mathrm{Wu} \mathrm{P}, \mathrm{Li}$ J. Treatment of branch retinal vein occlusion induced macular edema in treatment-naïve cases with a single intravitreal triamcinolone or bevacizumab injection. Chang Gung Med J. 2010;33(4):424-35.

16. Kansara V, Mitra A K. Evaluation of an ex vivo model implication for carrier-mediated retinal drug delivery. Curr Eye Res. 2006;31:415-26.

17. Cheruvu N P, Kompella U B. Bovine and porcine transscleral solute transport: influence of lipophilicity and the Choroid-Bruch's layer. Invest Ophthalmol Vis Sci. 2006;47:4513-22. 
18. Rosner B. Fundamentals of biostatistics. Belmont: Duxbury Press. 2006;557-581.

19. Liang K Y, Zeger S. Longitudinal data analysis using generalized linear models. Biometrika. 1986;73 (1):13-22.

20. Coscas G, Loewenstein A, Augustin A, Bandello F, Battaglia Parodi M, Lanzetta P, Mones J, de Smet M, Soubrane G, Staurenghi G. Management of retinal vein occlusion--consensus document. Ophthalmologica. 2011;226:4-28.

21. Ding X, Li J, Hu X, Yu S, Pan J, Tang S. Prospective study of intravitreal triamcinolone acetonide versus bevacizumab for macular edema secondary to central retinal vein occlusion. Retina. 2011;31:838-45.

22. Jue B Y, In R M, Chul L S, Lee D Y. Intravitreal triamcinolone acetonide versus bevacizumab therapy for macular edema associated with branch retinal vein occlusion. Graefes Arch. Clin. Exp. Ophthalmol. 2010;248:963-71.

23. Cunningham M A, Edelman J L, Kaushal S. Intravitreal steroids for macular edema: the past, the present, and the future. Surv Ophthalmol. 2008;53:139-49.

24. Lin J M, Chiu Y T, Hung P T, Tsai Y Y. Early treatment of severe cystoid macular edema in central retinal vein occlusion with posterior sub-tenon triamcinolone acetonide. Retina. 2007;27:180-9.

25. Anderson O A, Jackson T L, Singh J K, Hussain A A, Marshall J. Human transscleral albumin permeability and the effect of topographical location and donor age. Invest Ophthalmol Vis Sci. 2008;49:4041-5.

26. Ambati J, Gragoudas E S, Miller J W, You T T, Miyamoto K, Delori F C, Adamis A P. Transscleral delivery of bioactive protein to the choroid and retina. Invest Ophthalmol Vis Sci. 2000;41:1186-91.

27. Filippatos T D, Athyros V G, Elisaf M S. The pharmacokinetic considerations and adverse effects of DPP-4 inhibitors [corrected]. Expert Opin Drug Metab Toxicol. 2014;10:787-812.

28. Penfold P L, Wen L, Madigan M C, Gillies M C, King N J, Provis J M. Triamcinolone acetonide modulates permeability and intercellular adhesion molecule-1 (ICAM-1) expression of the ECV304 cell line: implications for macular degeneration. Clin Exp Immunol. 2000;121:458-65.

\section{Tables}

Due to technical limitations, all table files are only available for download from the Supplementary Files section.

\section{Figures}




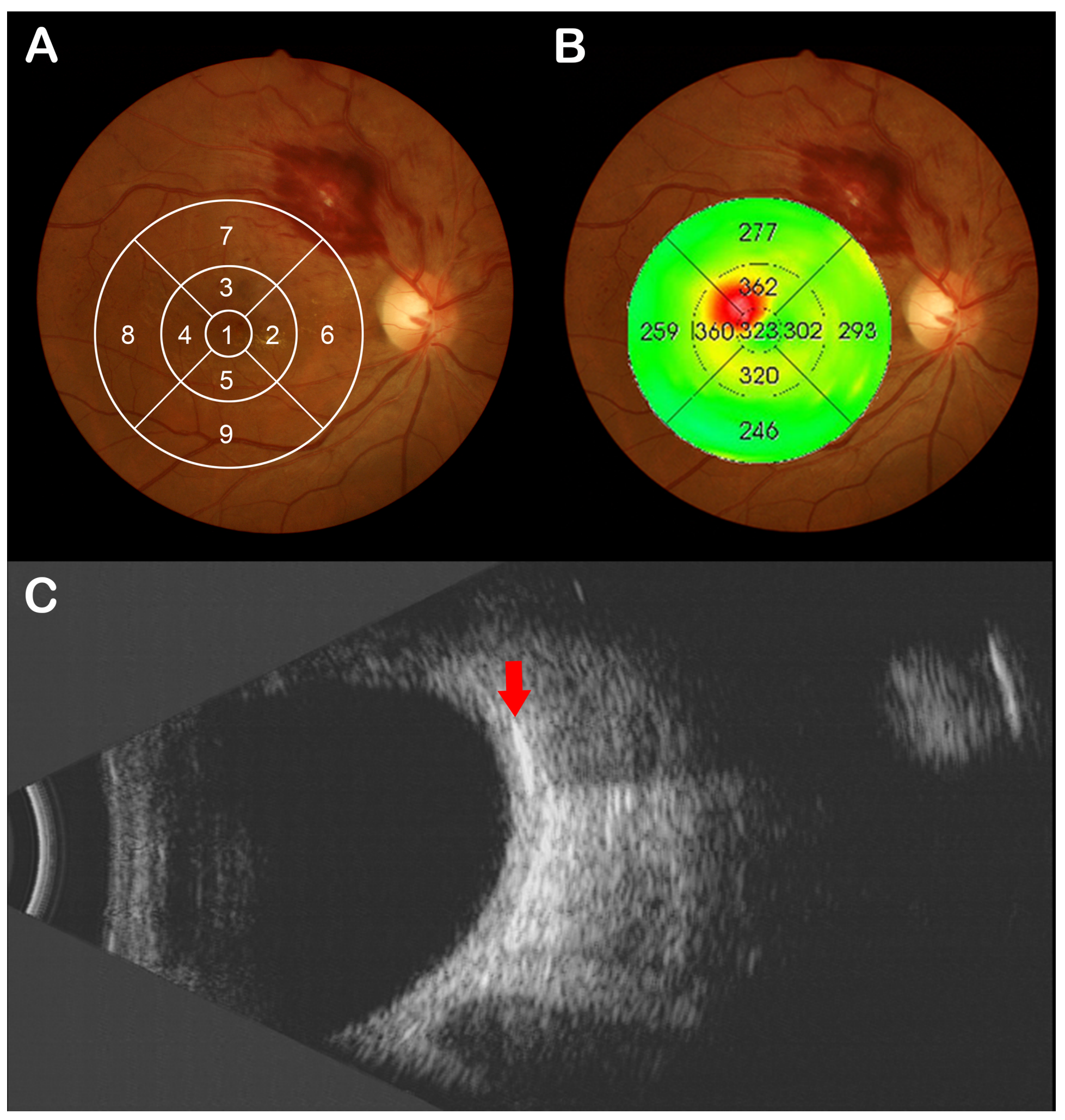

\section{Figure 1}

Diagram of macular zones for BRVO patients and B-ultrasonography image after PS-TA A: Diagram of macular zones; the 9 zones shown in the figure are centered at the macular fovea and numbered from 19. 1 represents the macular fovea (zone 1 with a diameter of $1000 \mu \mathrm{m}) ; 2-5$ represent the inner ring (diameter: 1000-3000 $\mu \mathrm{m}$ ), including 4 equal zones: temporal side, upper side, nasal side, and lower side; 6-9 represent the outer ring (diameter: $3000-6000 \mu \mathrm{m}$ ), including 4 equal zones: temporal side, upper side, 
nasal side, and lower side. B: OCT for macular area can generate 9 values corresponding respectively to the 9 zones. The OCT scanner scanned along the horizontal meridian lines of optic nerves with a scan length of $6 \mathrm{~mm}$. Each region corresponding numerical values; C: B-ultrasonography image obtained 5 days after PS-TA treatment; the arrow points to the drug residue posteriorly to the sclera in macular area, and sound shadow can be seen in the rear.

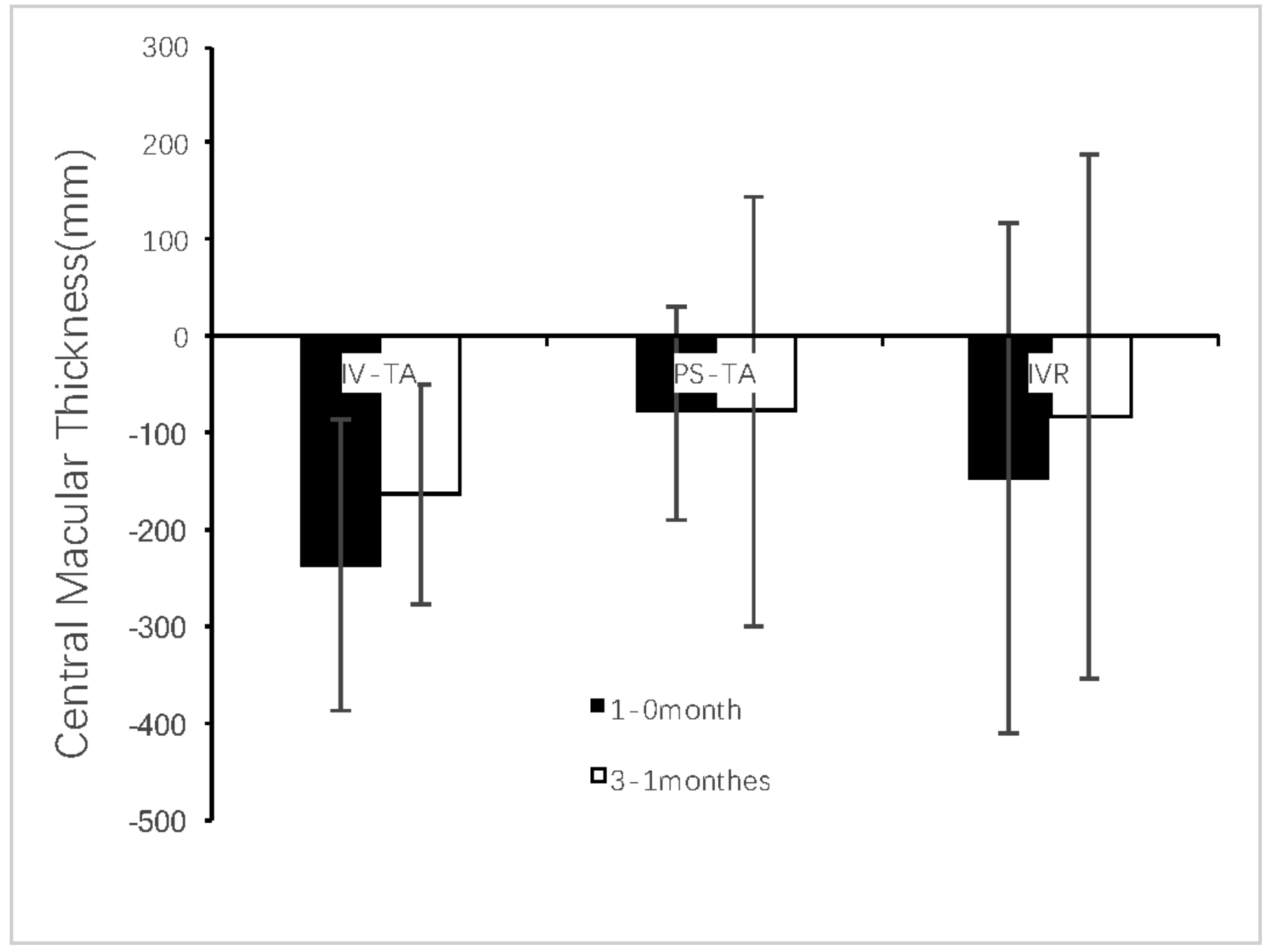

Figure 2

Changes of retinal thickness in macular fovea (zone 1) of BRVO with CME patients before and after treatments 


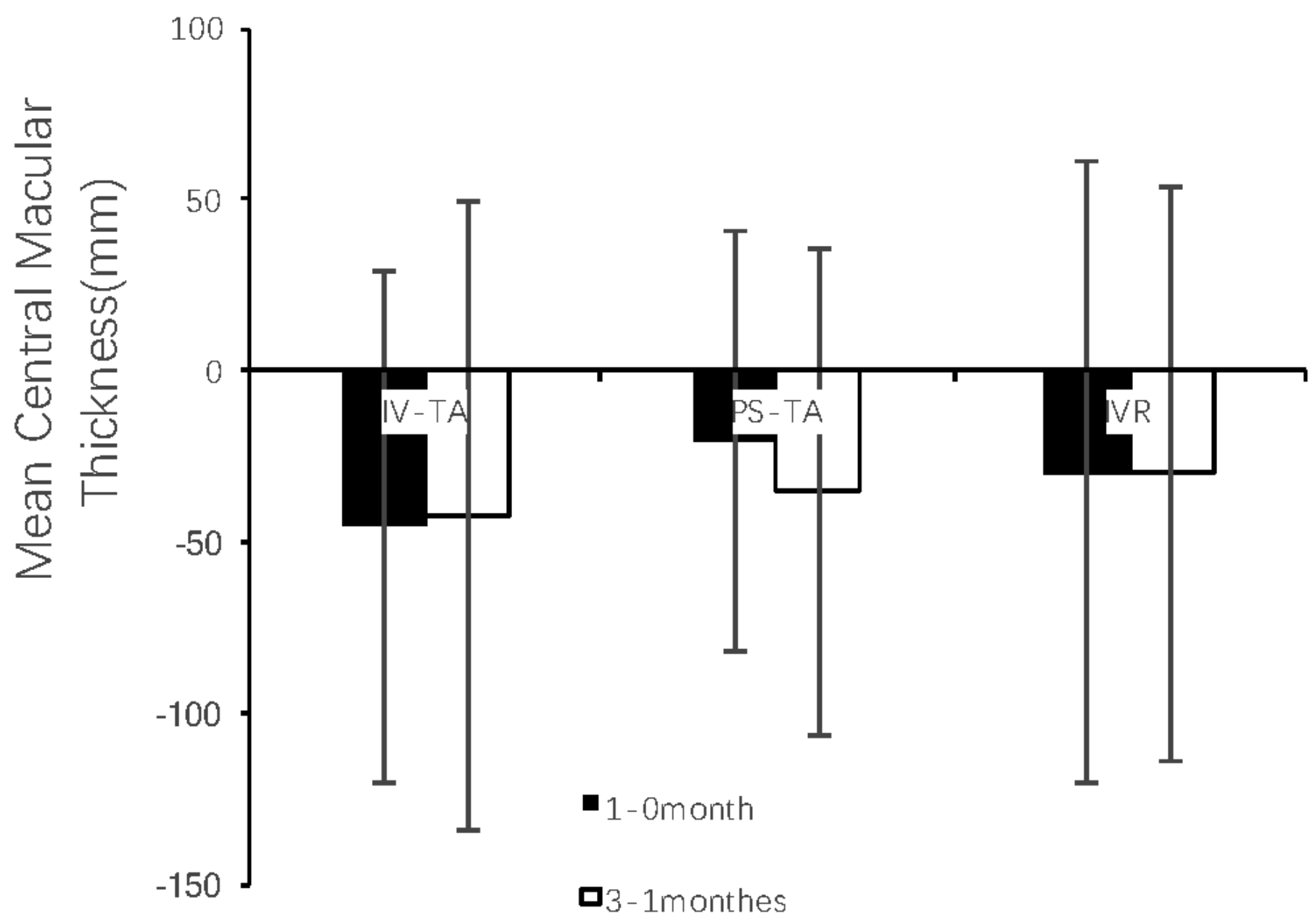

Figure 3

Changes of average thickness in macular area (1-9 zones) of BRVO with CME patients before and after treatments

\section{Supplementary Files}

This is a list of supplementary files associated with this preprint. Click to download.

- CONSORT2010Checklist.doc

- BRVOIhxsubmissionTables.docx 